Journal of Experimental and Clinical Medicine https://dergipark.org.tr/omujecm

Research Article

$\mathrm{J}$ Exp Clin Med

2021; 38(4): 525-528

doi: 10.52142 /omujecm.38.4.23

\title{
Histopathological effect of platelet-rich plasma on cranial dura mater defects
}

\author{
Kemal PAKSOY ${ }^{1, *}$ (i), Kerameddin AYDIN ${ }^{2}$ (D) \\ ${ }^{1}$ Department of Neurosurgery, Bahçelievler Memorial Hospital, İstanbul, Turkey \\ ${ }^{2}$ Department of Neurosurgery, Samsun Medical Park Hospital, Samsun, Turkey
}

\begin{abstract}
Received: 29.03 .2021
Accepted/Published Online: 30.03 .2021

$\bullet$

Final Version: 30.08.2021

Abstract

There are many new studies in the selection of materials used in dura material repair. Platelet has important function in hemostasis and coagulation. Also, activated platelets initiate the wound healing process. They provide regeneration of tissue with the appropriate type of tissue. In this experimental study, we used platelet-rich plasma (PRP) on dura mater defect due to these properties. Materials and method: Our study started after we got the approval of Ondokuz Mayis University Animal Experiments Local Ethics Committee. Thirty Wistar Albino female rats were used. The rats were divided into three groups, each one includes ten. Results: No significant difference was observed in the thickness and surface areas of dura mater in group 1 and group 2. Significant increase was observed in the thickness and surface areas of dura mater in the PRP applied group (Group 3) compared to other groups. New bone areas and new vasculature were observed more frequently in the PRP rooms group (Group 3). Statistical analysis of the data was done by using SPSS 21.0 for Mac (IBM Corporation) statistical package program and based on 0.05 significance level. A significant difference was observed between Group 1 and Group 3 ( $p>0.05$ ), a significant difference was observed between both Group 1 and Group 3 and between Group 2 and Group $3(p<0.05)$. Conclusion: Providing the regeneration of dura mater in defect situations will contribute to the protection of the barrier feature. In line with this idea, it is thought that PRP can be beneficial for us to reach this goal.
\end{abstract}

Keywords: cranial dura mater, histopathology, platelet-rich plasma

\section{Introduction}

The dura mater defect is a bothersome complication of neurosurgical practice. Dural tear can cause headache, cerebrospinal fluid (CSF) pseudocyst, meningitis, arachnoiditis, wound infection, and several other serious complications (1). These complications can be effectively decreased after the successful repair of dural tear (2). Dural repair is shown to decrease cystic cavity and connective tissue scar, increase tissue spare and reestablish function after dural injury in rats $(3,4)$. Thus, it is critical to repair it rapidly when dura mater tear arises. Suturing still remains the most common technique of dura mater repair. However, when dural tears are in moderately unreachable regions or enclosed by fragile dura mater, suture techniques are challenging to perform. This difficulty has led the neurosurgeons to support the use of several other methods. Cyanoacrylates, fibrin glue, and $\mathrm{CO}_{2}$ laser have all been preferred to achieve dura mater support $(5,6)$. Platelet-rich plasma (PRP) is a by-product of blood (plasma) comprising numerous bioactive aspects which are included in tissue restoration and has more than a few benefits compared with other dural repair techniques. PRP can be prepared from autologous blood and its clinical use causes no postoperative complications or adverse effects (7). PRP is comprised of various growth factors such as plateletderived, transforming-, insulin like-, fibroblast-, epithelial-, and vascular endothelial- growth factors (8). The presence of these growth factors is in charge for various factors involved in tissue regeneration such as angiogenesis, collagen production, increased cell proliferation, and induced cell differentiation (9). PRP-related products are used in several surgical interventions to stimulate the healing procedure after muscle, joint, and tendon injuries (10). There are numerous commercial products on the market for the repair of dura mater defects; however, the best technique of watertight dura mater closing has yet to be determined. In this animal study, we aimed to compare the histopathological effects of dural suture technique with combined suturing and topically applied PRP on dural healing in a rat model of dural injury and cerebrospinal fluid leakage.

\section{Materials and methods}

\subsection{Experimental design}

All animal studies were carried out with the approval of the Ondokuz Mayıs University Animal Care and Use Committee. Animals were housed at constant temperature $\left(20-22^{\circ} \mathrm{C}\right)$ and humidity 50-60\% with a 12-hour light and 12-hour dark cycle. They were allowed free access to water and standard rat chow. A total of 30 Wistar Albino female rats were allocated into three groups in random, which were control group (Group 1) $(\mathrm{n}=10)$, suturing group (Group 2) $(\mathrm{n}=10)$, and combined suturing and PRP group (Group 3) $(n=10)$.

Group 1 (Control group): Craniectomy + dural incision

Group 2 (only suturing group): Craniectomy + dural incision 
+ suturing (The dura was sutured with 10/0 Vicryl)

Group 3 (PRP group): Craniectomy + dural incision + suturing + PRP (The dura was sutured with 10/0 Vicryl and PRP was applied over sutured dura.)

\subsection{Surgical procedure}

We induced anesthesia via intramuscular administration of 80 $\mathrm{mg} / \mathrm{kg}$ ketamine hydrochloride (Ketalar, 5\% solution; Levent, Istanbul, Turkey) and $10 \mathrm{mg} / \mathrm{kg}$ xylazine (Rompun, $2 \%$ solution; Bayer, Leverkusen, Germany). After we shaved the parietal region of each rat, the surgical site was sterilized by povidone. Craniectomy ( $2 \mathrm{~cm}$ in diameter) was performed with a high-speed drill on the right parietal bone of all rats. The dura mater was kept intact during this procedure. The dura was then opened transversely and CSF leak was observed with the operating microscope. For Group 3, $1 \mathrm{cc}$ of fresh blood collected from the tail of the rats. The mixture was immediately centrifuged at $3000 \mathrm{rpm}$ for $8 \mathrm{~min}$. The top three-quarters of the supernatant, which consisted of the platelet-poor plasma, was aspirated and transferred to a new tube. The remaining fraction contained PRP. The surgical area was closed with $4 / 0$ silk and the rats were kept under conventional laboratory conditions. $100 \mathrm{mg} / \mathrm{kg}$ cefazolin (Cefazolin, Mustafa Nevzat, Istanbul, Turkey) was administered intramuscularly. All animals were sacrificed 21 days after the operation by decapitation. Both cerebral hemispheres and dura mater were totally excised. The samples were stored in $10 \%$ formaldehyde for histopathologic examinations at room temperature. The samples were embedded in paraplast and sectioned exhaustively. Coronal sections, $5 \mu \mathrm{m}$ thick, were cut at a $500 \mu \mathrm{m}$ sampling interval and stained with haematoxylin and eosin. The total volume of each dura mater was estimated using the Cavalieri principle.

\subsection{Cavalieri's principle}

The volume (V) of dura maters were estimated from "V = $\operatorname{ta}(\mathrm{p}) \Sigma \mathrm{P}$ " where $\mathrm{t}$ is the mean slice thickness $(0.5 \mathrm{~mm}), \mathrm{a}(\mathrm{p})$ the area associated with each point of the point-grid $(1 \mathrm{~mm} 2$ in this study; corrected for magnification), and $\Sigma \mathrm{P}$ is the total number of points hitting each dura mater. In the Cavalieri principle, a researcher obtains a data called coefficient of error $(\mathrm{CE})$ to evaluate the precision of estimates and to see the reliability of the point density of the grids and sectioning intervals. Since the cut surface areas of consecutive sections are not independent quantities, conventional statistical formulae of CE cannot be applied to determine the variance of their sum. Many of the researchers developed some formulas to obtain the $\mathrm{CE}$ for the Cavalieri estimation method. Those formulas not only provide the CE but also give information on the required number of slices and density of the point counting grid (Fig. 1).

\subsection{Statistical analysis}

Data were analyzed using the IBM Statistical Package for Social Sciences v13 (SPSS Inc., Chicago, IL, USA). The results for all items were expressed as mean $\pm \mathrm{SD}$, assessed within a $95 \%$ reliance and at a level of $p<0.05$ significance. Parametric tests were applied to data of normal distribution and nonparametric tests were applied to data of questionably normal distribution.
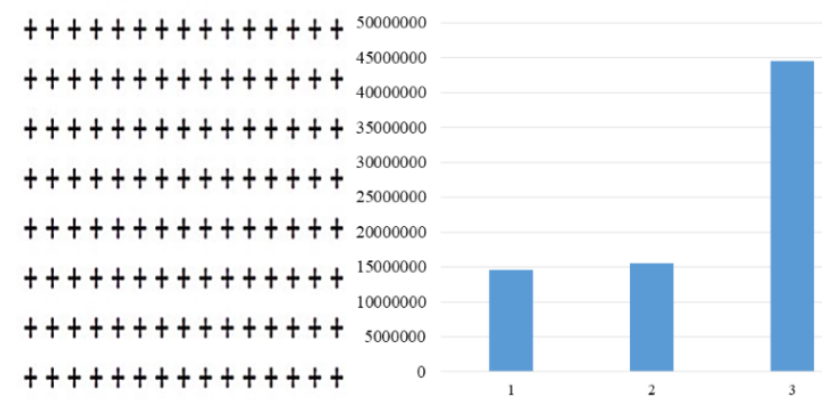

Fig. 1. Point counting grid used in the present study according to Cavalieri estimation method and graphical view

\section{Results}

No neurological deficits or wound infections were developed. In Group 1, regularly organized collagenous fibers were observed. In Groups 2 and 3, heterogeneous and regularly organized arrays of collagenous fibers were observed. In Group 3 (PRP Group), a significant increase in the volume of dura mater was determined when compared with other groups $(p<0.05)$. Areas of neogenesis and neovascularization were observed more frequently in the regions near the defect in group 3. On these regions, markedly higher osteoblastic activity was observed due to more intense peripheral infiltration tissue, while inflammation was less intense on the regions in the vicinity of neo-osteogenic areas. Newly formed bone tissue around the intact bone was extending into the defective area. In all experimental groups, new areas of bone, and vascularizations on the edges of the defective areas attracted our attention. In all groups patchy areas of narrowing were observed on arachnoid membranes. In all groups any macroscopic changes were not observed in pia mater (Fig. 2). Mean volumes of dura mater in Groups 1, 2 and 3 were calculated as 14616000 (455705) micrometer cubed $\left(\mu \mathrm{m}^{3}\right), 15558400$ (264649) $\mu^{3}$ and 44515200 (327769) $\mu \mathrm{m}^{3}$, respectively. Any statistically significant difference was not found between Groups 1 , and 2 ( $\mathrm{p}>0.05$ ), while a significant difference was observed between Groups 1, and 3, and also between Groups 2 and $3(\mathrm{p}<0.05)$ (Table 1).

Table 1. Statistical data table

\begin{tabular}{|c|c|c|c|c|c|c|}
\hline Group & Estimated Area $\mu \mathrm{m}^{2}$ & $\begin{array}{l}\text { Estimated/Volume } \\
\mu \mathrm{m}^{3}\end{array}$ & $\begin{array}{l}\text { Corrected Volume- } \\
\mu \mathrm{m}^{3}\end{array}$ & Error Coefficient & $\begin{array}{l}\text { Section Shear } \\
\text { Thickness }(\mu \mathrm{m})\end{array}$ & Census \\
\hline 1.Group & 487200 & 14616000 & 14048000 & 0.064 & 10 & 1218 \\
\hline 2.Group & 534000 & 16020000 & 15368000 & 0.064 & 10 & 1335 \\
\hline 3.Group & 1507200 & 45216000 & 43464000 & 0.048 & 10 & 3768 \\
\hline
\end{tabular}




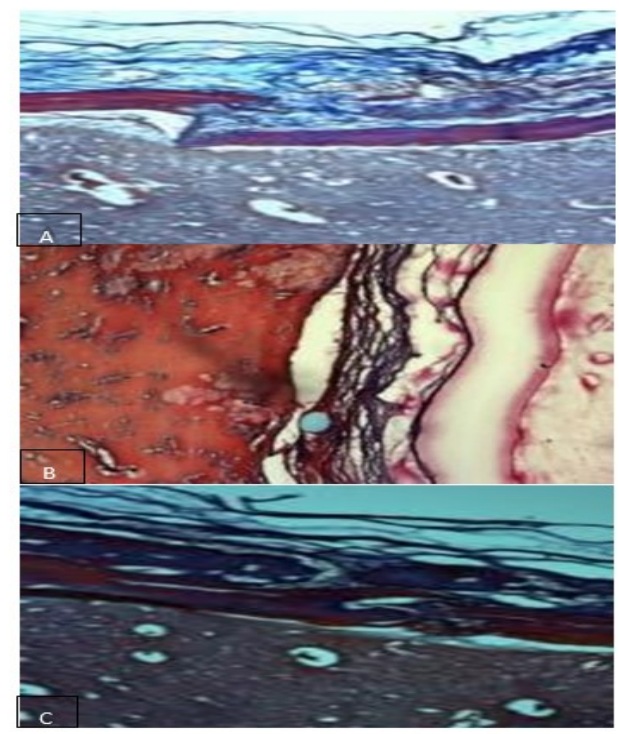

Fig. 2. The samples were embedded in paraplast and sectioned exhaustively. Coronal sections, $5 \mu \mathrm{m}$ thick, were cut at a $500 \mu \mathrm{m}$ sampling interval and stained with haematoxylin and eosin. (A) Group 1 (Control group): Craniectomy + dural incision (B) Group 2 (only suturing group): Craniectomy + dural incision + suturing (C) Group 3 (PRP group): Craniectomy + dural incision + suturing + PRP. A. In Group 1, regularly organized collagenous fibers were observed. B. In Groups 2, heterogeneous and regularly organized arrays of collagenous fibers were observed. C. In Groups 3, heterogeneous and regularly organized arrays of collagenous fibers were observed. Areas of neogenesis and neovascularization were observed more frequently in the regions near the defect in group 3. On these regions, markedly higher osteoblastic activity was observed due to more intense peripheral

\section{Discussion}

The dura mater is a collagenous tissue including various fibroblasts, collagen and elastic fibers. The dura mater may be damaged by trauma or surgery. Dural repair has recently garnered increased attention because CSF leakage leads to various secondary complications. Herein, we aimed to compare dural suture technique with combined suturing and PRP on the dural volume. Many neurosurgical interventions necessitate dural opening to maintain entree to central nervous system; besides, unplanned dura mater tears may arise frequently. Several harmful concerns of imperfectly treated dural CSF leaks are described (11). Collection of fluid avoids appropriate wound healing and can lead to incision infection and/or breakdown $(12,13)$. A constant leak of CSF may also lead to pseudo meningocele which may develop nerve roots trapping and neurological symptoms such as cranial nerve palsies, particularly of cranial nerve VI, which manifests as strabismus (14). The principles for the ultimate tissue adhesive comprise anticipation of CSF leak, no increase in infection rate, minimal foreign body or allergic reaction, cost effective, simplicity of application and availability. Consequently, the most essential characteristic of management of a CSF leak is avoidance. Dural suture is a simple and reliable method extensively preferred in cranial surgery $(15,16)$. Watertight suturing of a dural defect is not an easy surgical procedure in the deep, narrow surgical fields. After the hematological mediators were discovered, they were revealed to help wound healing. These mediators have proliferative cellular effects, which is also true for PRP. Farrag et al. applied PRP in cases with facial nerve injuries, and reported its favorable effects on the nerve regeneration (17). Sariguney et al. applied PRP in cases with sciatic nerve injuries, and reported its favorable contribution to axonal regeneration (18). In the present study, heterogeneous and regularly organized arrays of collagenous fibers were observed in suturing groups (Groups 2 and 3). Elgazzar et al. used cyanoacrylate sealants, and PRP in combination in cases with end-to-end sciatic nerve anastomosis, and reported successful anastomosis (19). Lichtenfels et al. used autologous nerve grafts in combination with PRP in anastomosis of sciatic nerves and published their successful results (20). Ding et al. and $\mathrm{Wu}$ et al., detected prominent contribution of PRP to axonal remyelination in cases with cavernous nerve injuries $(21,22)$. Cho et al. used PRP and mesenchymal stem cells in combination in cases with facial nerve injuries, and revealed its favorable effect on regeneration (23). Zheng et al. reported favorable effects of PRP on proliferation, and migration of Schwann cells, and also synthesis of neurotrophic factors (24). Zhao et al. used PRP, brain-derived neurotrophic factor, and stromal cells of bone marrow in combination, and reported functional improvement through achievement of axonal remyelination in cases with spinal cord injuries (25). In the present study, a significant increase in the volume of dura mater was determined in Group 3 (PRP Group) when compared with other groups. Furthermore, in Group 3 neogenesis, neovascularization, and osteoblastic activity were more frequently encountered, while inflammation was less intense in the regions near the defect when compared with other groups. Animal studies showed that experimental animals infrequently develop meningitis regardless of marked CSF leakage and histologically confirmed inflammation. CSF leak in humans is challenging to avoid and also treat, and leads to morbidity and mortality after some special neurosurgical approaches (26). That is why a persistent dural seal makes a clinically important difference in the prognosis. One of the limitations of this study is the short follow up of eight weeks. However, this time period is the equivalent of 27 human months (27). Although we still consider our results as preliminary, they warrant a larger comprehensive study, one which would include more rats in order to evaluate more precisely the role of platelet rich plasma in dural tear pathogenesis.

When all these findings are taken into consideration, according to our study, PRP conferred a marked benefit in the reconstruction of dura mater defects. We conclude that PRP can be safely used for the repair of dura mater defects. Its neo-osteogenic activity also signifies its significant effect on bone defects. 


\section{Conflict of interest}

The authors have no conflict of interest declaration.

\section{Acknowledgments}

There is no financial support for our study.

\section{References}

1. Sugawara T, Itoh Y, Hirano Y, Higashiyama N, Shimada Y, Kinouchi $\mathrm{H}$, et al. Novel dural closure technique using polyglactin acid sheet prevents cerebrospinal fluid leakage after spinal surgery. Neurosurgery. 2005; 57(4 Suppl):290-4; discussion 2904. doi: 10.1227/01.neu.0000176410.65750.c0.

2. Wang JC, Bohlman HH, Riew KD. Dural tears secondary to operations on the lumbar spine. Management and results after a two-year-minimum follow-up of eighty-eight patients. J Bone Joint Surg Am. 1998; 80(12):1728-32. doi: 10.2106/00004623199812000-00002.

3. Wang JC, Bohlman HH, Riew KD. Dural tears secondary to operations on the lumbar spine. Management and results after a two-year-minimum follow-up of eighty-eight patients. J Bone Joint Surg Am. 1998; 80(12):1728-32. doi: 10.2106/00004623199812000-00002

4. Liang H, Li C, Gao A, Liang P, Shao Y, Lin T, et al. Spinal duraplasty with two novel substitutes restored locomotor function after acute laceration spinal cord injury in rats. J Biomed Mater Res B Appl Biomater. 2012; 100(8):2131-40. doi: 10.1002/jbm.b.32778.

5. Foster LJ, Karsten E. A chitosan based, laser activated thin film surgical adhesive, 'SurgiLux': preparation and demonstration. J Vis Exp. 2012; (68):3527. doi: 10.3791/3527.

6. Ozisik PA, Inci S, Soylemezoglu F, Orhan H, Ozgen $\mathrm{T}$. Comparative dural closure techniques: a safety study in rats. Surg Neurol. 2006; 65(1):42-7; discussion 47. doi: 10.1016/j.surneu.2005.04.047.

7. Kotsovilis S, Markou N, Pepelassi E, Nikolidakis D. The adjunctive use of platelet-rich plasma in the therapy of periodontal intraosseous defects: a systematic review. J Periodontal Res. 2010; 45(3):428-43. doi: 10.1111/j.1600-0765.2009.01236.x.

8. Andia I, Sánchez M, Maffulli N. Joint pathology and platelet-rich plasma therapies. Expert Opin Biol Ther. 2012; 12(1):7-22. doi: $10.1517 / 14712598.2012 .632765$.

9. Arora NS, Ramanayake T, Ren YF, Romanos GE. Platelet-rich plasma: a literature review. Implant Dent. 2009; 18(4):303-10. doi: 10.1097/ID.0b013e31819e8ec6.

10. Gundersen HJ, Jensen EB. The efficiency of systematic sampling in stereology and its prediction. J Microsc. $1987 \mathrm{Sep} ; 147(\mathrm{Pt}$ 3):229-63. doi: 10.1111/j.1365-2818.1987.tb02837.x.

11. Hutter G, von Felten S, Sailer MH, Schulz M, Mariani L. Risk factors for postoperative CSF leakage after elective craniotomy and the efficacy of fleece-bound tissue sealing against dural suturing alone: a randomized controlled trial. J Neurosurg. 2014; 121(3):735-44. doi: 10.3171/2014.6.JNS131917.

12. Luszczyk MJ, Blaisdell GY, Wiater BP, Bellabarba C, Chapman JR, Agel JA, et al. Traumatic dural tears: what do we know and are they a problem? Spine J. 2014; 14(1):49-56. doi: 10.1016/j.spinee.2013.03.049.

13. $\mathrm{Yu}$ F, Wu F, Zhou R, Guo L, Zhang J, Tao D. Current developments in dural repair: a focused review on new methods and materials. Front Biosci (Landmark Ed). 2013 Jun 1; 18:133543. doi: $10.2741 / 4182$.

14. Joo JD, Yoon $\mathrm{SH}$, Kim KJ, Jahng TA, Kim HJ. Isolated abducens nerve palsy due to cerebrospinal fluid leakage following lumbar discectomy: a rare clinical entity. Eur Spine J. 2013 May;22 Suppl 3(Suppl 3): S421-3. doi: 10.1007/s00586$012-2545-\mathrm{z}$.

15. Osbun JW, Ellenbogen RG, Chesnut RM, Chin LS, Connolly PJ, Cosgrove GR, et al. A multicenter, single-blind, prospective randomized trial to evaluate the safety of a polyethylene glycol hydrogel (Duraseal Dural Sealant System) as a dural sealant in cranial surgery. World Neurosurg. 2012; 78(5):498-504. doi: 10.1016/j.wneu.2011.12.011.

16. Von der Brelie C, Soehle M, Clusmann HR. Intraoperative sealing of dura mater defects with a novel, synthetic, self adhesive patch: application experience in 25 patients. $\mathrm{Br} \mathrm{J}$ Neurosurg. 2012 Apr;26(2):231-5. doi: $10.3109 / 02688697.2011 .619597$.

17. Farrag TY, Lehar M, Verhaegen P, Carson KA, Byrne PJ. Effect of platelet rich plasma and fibrin sealant on facial nerve regeneration in a rat model. Laryngoscope. 2007; 117(1):157-65. doi: 10.1097/01.mlg.0000249726.98801.77.

18. Sariguney Y, Yavuzer R, Elmas C, Yenicesu I, Bolay H, Atabay $\mathrm{K}$. Effect of platelet-rich plasma on peripheral nerve regeneration. J Reconstr Microsurg. 2008; 24(3):159-67. doi: 10.1055/s-2008-1076752.

19. Elgazzar RF, Mutabagani MA, Abdelaal SE, Sadakah AA. Platelet rich plasma may enhance peripheral nerve regeneration after cyanoacrylate reanastomosis: a controlled blind study on rats. Int J Oral Maxillofac Surg. 2008; 37(8):748-55. doi: 10.1016/j.ijom.2008.05.010.

20. Lichtenfels M, Colomé L, Sebben AD, Braga-Silva J. Effect of Platelet Rich Plasma and Platelet Rich Fibrin on sciatic nerve regeneration in a rat model. Microsurgery. 2013; 33(5):383-90. doi: 10.1002/micr.22105.

21. Ding XG, Li SW, Zheng XM, Hu LQ, Hu WL, Luo Y. The effect of platelet-rich plasma on cavernous nerve regeneration in a rat model. Asian J Androl. 2009; 11(2):215-21. doi: 10.1038/aja.2008.37.

22. Wu CC, Wu YN, Ho HO, Chen KC, Sheu MT, Chiang HS. The neuroprotective effect of platelet-rich plasma on erectile function in bilateral cavernous nerve injury rat model. J Sex Med. 2012 Nov;9(11):2838-48. doi: 10.1111/j.1743-6109.2012.02881.x.

23. Cho HH, Jang S, Lee SC, Jeong HS, Park JS, Han JY, et al. Effect of neural-induced mesenchymal stem cells and plateletrich plasma on facial nerve regeneration in an acute nerve injury model. Laryngoscope. 2010; 120(5):907-13. doi: 10.1002/lary.20860.

24. Zheng C, Zhu Q, Liu X, Huang X, He C, Jiang L, et al. Effect of platelet-rich plasma (PRP) concentration on proliferation, neurotrophic function and migration of Schwann cells in vitro. J Tissue Eng Regen Med. 2016; 10(5):428-36. doi: 10.1002/term. 1756 .

25. Zhao T, Yan W, Xu K, Qi Y, Dai X, Shi Z. Combined treatment with platelet-rich plasma and brain-derived neurotrophic factoroverexpressing bone marrow stromal cells supports axonal remyelination in a rat spinal cord hemi-section model. Cytotherapy. 2013; 15(7):792-804. doi: 10.1016/j.jcyt.2013.04.004.

26. Shonka DC Jr, Potash AE, Jameson MJ, Funk GF. Successful reconstruction of scalp and skull defects: lessons learned from a large series. Laryngoscope. 2011; 121(11):2305-12. doi: 10.1002/lary.22191.

27. Christou NV, Look D, Maclean LD. Weight gain after short- and long-limb gastric bypass in patients followed for longer than 10 years. Ann Surg. 2006; 244(5):734-40. 